ISSN: 2162-3104 Print/ ISSN: 2166-3750 Online

Volume 8, Issue 4 (2018), pp. 1549-1568

(C) Journal of International Students

http://jistudents.org/

doi: 10.5281/zenodo.1467809

\title{
The Outcomes of the Community Cooking Workshops for International Students at Simon Fraser University
}

\author{
Gabriella Luongo \\ Simon Fraser University, Canada \\ Rochelle Tucker \\ Simon Fraser University, Canada \\ Crystal Hutchinson \\ Simon Fraser University, Canada \\ Rosie Dhaliwal \\ Simon Fraser University, Canada
}

\begin{abstract}
The Community Cooking Workshops (CCWs) are free, bi-weekly, hands-on, cooking and nutrition education workshops for international students facilitated by the Simon Fraser University (SFU) Peer Health Educators (PHEs). The impacts of the CCWs on international student nutrition knowledge, behavior change, and social outcomes were examined. Data was collected and coded from five Spring 2016 CCWs through workshop evaluation forms ( $n=87,93 \%$ response rate) and nine semi-structured interviews with international student participants. Participants reported changes in eating behavior and knowledge (all food should be consumed in moderation, with variety) and improved sense of community. Future research should assess the long-term impacts of cooking workshops in postsecondary institutions on diet quality, cooking skills, social connectedness, and international student wellbeing.
\end{abstract}

Keywords: cooking, attitudes practice, health promotion, mental health, students 
Adjusting to the academic and social demands of a post-secondary institution can be difficult for international students (Almohanna, Conforti, Eigel, \& Barbeau, 2015; Vafors Fritz, Chin, \& DeMarinis, 2008). While domestic post-secondary students may be required to learn how to prepare meals for themselves, and manage their own finances, international students are required to perform these tasks in a new country with an unfamiliar language, all while adapting to foreign customs (Almohanna, Conforti, Eigel, \& Barbeau, 2015; Smith \& Khawaja, 2011). These added pressures can result in increased anxiety, stress, sleep disorders, and depression among the international student population, who in comparison to their domestic counterparts, are at a greater risk of developing mental health problems (Almohanna, Conforti, Eigel, \& Barbeau, 2015; Fritz, Chin, \& DeMarinis, 2008). Given that international students are at increased levels of stress in comparison to their domestic counterparts (Almohanna, Conforti, Eigel, \& Barbeau, 2015), and the implications of stress on the eating patterns of postsecondary students (Alakaam, Castellanos, Bodzio \& Harrison, 2016), supporting international students' eating patterns should be of prime importance. Therefore, interventions designed to improve the nutritional practices of post-secondary international students, such as cooking workshops, should be explored.

\section{LITERATURE REVIEW}

Healthy eating patterns play a key role in the academic success of postsecondary students, yet post-secondary students consume more high-fat, high-calorie foods as compared to fruits and vegetables on a daily basis (Versaevel, 2014). The 2016 Canadian National College Health Assessment (NCHA) found that only $10 \%$ of students met the recommendations from the Eating Well with Canada's Food Guide for fruit and vegetable consumption (Versaevel, 2014). In the 2016 NCHA, 46.2\% of postsecondary students reported more than average stress, and $14.4 \%$ indicated they experienced tremendous stress (ACHA, 2016). High perceived stress in post-secondary students is associated with increased snack food, carbohydrate, and sugar consumption, and decreased fruit and vegetable consumption (Alakaam, Castellanos, Bodzio \& Harrison, 2016). Moreover, studies have consistently reported associations between increased "unhealthy" diets, those high in fat and processed sugar, with increased diagnoses of depression and anxiety disorders (Dietitians of Canada, 2012).

Post-secondary institutions can provide an optimal setting for health education. For international students in a new environment, the post- 
secondary setting may be one of the first places they acquaint themselves with upon arrival and therefore, is a key context for accessing health information. Using the post-secondary environment to facilitate nutrition interventions could provide an opportunity for international students to receive accurate nutrition and health information and serve as a first point of contact to connect students with other on-campus health and non-health related resources. By providing international students with tools and information to improve their nutrition knowledge and cooking self-efficacy, this may encourage international students to cook for themselves and avoid commercially prepared meals, thereby improving their diet quality (Thorpe, Kestin, Riddell, Keast \& McNaughton, 2013).

\section{Interventions for Improving Nutritional Practices Among Post- Secondary Students}

A review of recent literature on post-secondary nutrition interventions provides four nutrition interventions targeted at improving the nutritional practices of post-secondary students: cooking shows, cooking classes, cooking demonstrations, and dinner groups (Ball \& Brown, 2012; Clifford, Anderson, Auld \& Champ, 2009; Lawe, 2013; Levy \& Auld, 2004).

Clifford, Anderson, Auld and Champ (2009), assessed the outcomes of a randomized control trial Social Cognitive Theory based cooking show for post-secondary students. While the study found significant improvements in knowledge about fruit and vegetable consumption, this knowledge did not translate into health behaviours (Clifford, Anderson, Auld \& Champ, 2009). Similarly, Levy and Auld's (2004) assessment of cooking classes and cooking demonstrations by trained nutrition professionals found statistical improvement in cooking attitudes and cooking self-efficacy for students who participated in the cooking classes, however there were no statistical differences in cooking related knowledge and behaviours (Clifford, Anderson, Auld \& Champ, 2009).

A third study by Ball and Brown (2012) assessed dinner groups on postsecondary campuses. Dinner groups were defined as a "group of three people or more cooking for one another (or together) and eating together at least four times a week" (Ball \& Brown, 2012). Students reported that participating in dinner groups provided students a social outlet, allowed students to make new friends, increase their cooking confidence, reduced cooking time, improved cost effectiveness for students and helped students consume more healthful and varied foods. The study found that the value of the dinner groups increased as socialization was incorporated into mealtimes (Ball \& Brown, 2012). Students reported that the negative aspects of the 
dinner groups included increased time spent on days when the student cooked, unreliable group members, worrying about people liking the food, and the stresses involved in adhering to a cooking schedule (Ball \& Brown, 2012). However, the literature concluded that the benefits of participating in a dinner group far outweighed the challenges (Ball \& Brown, 2012).

A final study evaluated student facilitated cooking classes for postsecondary students in the United Kingdom (Lawe, 2013). This program was offered for four weeks as a pilot project (Lawe, 2013). All participants reported that their cooking skills improved and felt more confident in cooking; however only $50 \%$ indicated that they would continued to cook healthy food after the session (Lawe, 2013). No evaluation of short- or longterm nutritional changes was assessed (Lawe, 2013).

Only in the Lawe (2013) study were student leaders used to facilitate the workshops. Using student leaders could be effective in the delivery of nutrition interventions. Peer Health Educators (PHEs) play a valuable role in decreasing the prevalence of high risk behaviors such as unhealthy alcohol and food consumption among post-secondary students and can act as credible role models for the student populations (White, Park, Israel \& Cordero, 2009). Research suggests that students who come in contact with PHEs report decreased "fat talk" and unhealthy weight management techniques (White, Park, Israel \& Cordero, 2009). Additionally, social support networks moderate the relationship between acculturative stress and mental health symptoms for international students, where increased social support networks improve sociocultural adjustment and decrease acculturative stress (Smith \& Khawaja, 2011; Zhang \& Goodson, 2010). Therefore, PHEs could be beneficial in providing initial social support to ease the transition to a new environment for international students. The current literature does not provide any research that focuses on nutrition interventions tailored to the international student population. The cooking workshop intervention evaluated presents an example of a sustainable intervention that uses peer facilitators to improve international students' nutrition behaviors and knowledge, and social connection.

\section{BACKGROUND}

\section{Simon Fraser University}

Simon Fraser University is Canada's leading comprehensive university established in 1965. Located in the Province of British Columbia, SFU has three campuses situated in adjacent municipalities. The main campus is located in the City of Burnaby, and two others in the City of Vancouver and 
Surrey. SFU is home to over 25,000 undergraduate, full-time and part-time students and 4,000 graduate students in a variety of faculties including Business, Health Sciences, Arts and Social Sciences, Applied Science, Science, Education, Environment, Communication, Art and Technology.

As of Fall 2017, nearly 5,000 undergraduate international students were registered at SFU (19.4\% of the total undergraduate population), while over 1,000 graduate students were international $(28.3 \%$ of the total graduate population) (SFU Institutional Research and Planning [IRP], 2017). The SFU international student population is growing yearly, with a majority of international students holding Chinese or Indian citizenship (SFU IRP, 2017).

\section{The Simon Fraser University Peer Health Educators}

The SFU Peer Health Educators are a group of 20 student volunteers trained and supervised by the Health Promotion team in Health and Counselling Services located at the Burnaby campus. The Peer Health Educators lead and evaluate health education outreaches across all three SFU campuses to enhance student health and well-being while addressing specific topics including resilience, stress management, sexual health, body image, sleep, active living and nutrition.

\section{The Community Cooking Workshops}

The SFU Peer Health Educators and Health Promotion team initiated the Community Cooking Workshops (CCWs) in September 2013, in consultation with SFU International Services for Students. The CCWs were initially offered once per semester and focused on one snack or meal item but in September 2015, as a result of a Changing the Culture of Substance Use (CCSU) mini grant from the Canadian Mental Health Association, the workshops were offered once per month as dinner workshops. In spring 2016, the Health Peers expanded the program to deliver five bi-weekly cooking workshops.

With the help of the CCSU mini-grant, the PHEs were able to purchase cooking supplies such as cutting boards and measuring spoons for 20 participants, as well as cooking utensils and appliances such as a blender, pots, and pans. Nesters Market, a local grocery store, sponsored the ingredients for the CCWs. SFU Health and Counselling Services covered miscellaneous ingredients and supplies, while SFU Residence and Housing provided free use of the kitchen space to facilitate these Workshops at the Burnaby campus. 
The cooking workshops are promoted on social media by SFU International Services for Students, the SFU Peer Health Educators, SFU Health and Counselling Services, and SFU Health Promotion. An online survey is used for registration, which often reaches full capacity within 48 hours. The dinner menus and nutrition presentations are created by the SFU Peer Health Educators in consultation with SFU Health Promotion.

There are two primary objectives of the Community Cooking Workshops.

1. To equip SFU international students with the food preparation skills and increase awareness of healthy eating and associated behaviours.

2. To increase social connection among SFU international students.

The workshops are free and provide an opportunity for up to 20 international student participants to learn valuable cooking skills while forming meaningful social connections in a safe and supportive setting. The workshops are aimed at enhancing cooking competence among the international student population through hands-on workshops where students work together with guidance from Peer Health Educators to follow a recipe and prepare a meal together. During each workshop, a 10-minute presentation or facilitated discussion is led by the PHE team focused on health topics including resilience, stress management, body image, healthy and mindful eating practices, and eating on a budget.

\section{Study Goals}

There has been limited research examining the impact of nutrition-based interventions for post-secondary international student populations. The goal of this research project was to understand the outcomes of the CCWs for international student participants within the context of SFU. The data collected will be used to inform future outreaches for international students and cooking workshops led by the PHEs, and other initiatives for international students organized by SFU Health Promotion, Health and Counselling Services and SFU International Services for Students.

\section{RESEARCH METHOD}

\section{Participants}

Participants in this study included undergraduate and graduate students at SFU who attended at least one of the five CCWs from January to April 
2016. Upon workshop registration, participants completed a brief survey assessing demographics. In total, 63 unique students participated in at least one of the five workshops, which resulted in 87 cooking workshop feedback forms completed of the 94 overall number of participants who attended the program in spring 2016 (includes students who attended more than one workshop). Table 1 presents a breakdown of the study population who attended the cooking workshops. Students were not asked about their sex upon workshop registration.

Table 1. Demographics of students who participated in at least one CCW in Spring $2016(n=63)$.

\begin{tabular}{lc} 
Demographic & $\begin{array}{c}\text { Number of participants } \\
\text { (\% of total) }\end{array}$ \\
\hline Length at SFU if an international student \\
(semesters) \\
$\leq 1$ semester & \\
$2-4$ & $19(30 \%)$ \\
$\geq 5$ & $15(24 \%)$ \\
Non-response & $14(22 \%)$ \\
Non-international students & $5(8 \%)$ \\
Area of origin & $10(16 \%)$ \\
South Asia & \\
Middle East & $5(8 \%)$ \\
Europe & $2(3 \%)$ \\
China & $8(13 \%)$ \\
Other South East Asian countries & $20(32 \%)$ \\
Africa & $13(21 \%)$ \\
United States & $2(3 \%)$ \\
South America & $2(3 \%)$ \\
Canada (local) & $1(1 \%)$ \\
Non-response & $10(16 \%)$ \\
Current living arrangements & $0(0 \%)$ \\
Off campus & \\
Residence & $29(46 \%)$ \\
UniverCITY (on campus, not & $27(43 \%)$ \\
Residence housing) & $4(6 \%)$ \\
At home & \\
Degree type & $3(5 \%)$ \\
Undergraduate & \\
Graduate & $49(78 \%)$ \\
Student type & $14(22 \%)$ \\
International & \\
Domestic & $53(84 \%)$ \\
\hline & $10(16 \%)$ \\
\hline
\end{tabular}


Nine international students who participated in at least one cooking workshop in the spring 2016 semester were interviewed. Of the nine students, there were four undergraduate students, four Master's students, and one PhD student. The majors of these students were Criminology, Education, Psychology, Computing Science, Finance, and Health Sciences. Students were from Tanzania, China, the United States, Nigeria, and Russia. One male and eight female students were interviewed.

\section{Data Collection}

Following each workshop from January to April 2016, anonymous evaluation forms were distributed to participants to collect information assessing key learnings including changes in knowledge and motivation to change behaviour as a result of the workshop, social connections, and to gain feedback on workshop delivery. These evaluation forms were designed by SFU Health Promotion with feedback from the PHEs. The forms asked questions on a five-point Likert scale (strongly agree, agree, neutral, disagree, strongly disagree). The feedback forms asked questions about students cooking confidence, motivation or intention to improve their nutrition patterns, and knowledge about healthy eating as a result of participating in the workshop. The open-ended questions on the forms asked students about what they liked and what could be improved about workshop delivery.

Emails were sent to all spring $2016 \mathrm{CCW}$ attendees inviting international student participants to participate in a 60-minute semistructured interview. Nine international students responded to the email request and were interviewed by the primary investigator. Nine semistructured interviews spanning 47-81 minutes were conducted with international students who had participated in at least one cooking workshop in spring 2016 in a quiet room at SFU. All interviews were conducted in English and no interpreters were present. Students were asked about how the workshops impacted their nutritional knowledge and cooking abilities, food purchasing and eating habits, and perceived barriers as international students in terms of maintaining a balanced diet in university. The interviews were audio recorded, transcribed and coded. Each participant was given a \$20 $(\mathrm{Cdn})$ gift card as an honorarium.

Ethics approval was obtained from the SFU Office of Research Ethics to use the feedback forms for secondary data analysis and to conduct the interviews. Interview participants provided written consent for their information to be used in this study. 


\section{Analytic Approach}

Responses to the open-ended feedback form questions were coded for common themes, while the Likert scale questions were analyzed using a quantitative analysis through RStudio. For each Likert scale question, students' responses of "strongly agree" or "agree" were combined.

The semi-structured interviews were transcribed verbatim and coded by the primary investigator using a variety of themes that emerged in the data. The coding was checked for accuracy and consistency by the faculty supervisor.

Additional information gathered through the semi-structured interviews such as eating habits, and barriers to healthy eating, were also coded and analyzed, and are presented in the results section.

\section{RESULTS}

\section{Quantitative Analysis: Participant Feedback Forms}

In spring 2016, a total of five cooking workshops were held with 63 unique participants. Of the 94 feedback forms collected over the five workshops, 87 completed feedback forms were analyzed with a 93\% response rate. Ten $(16 \%)$ were identified as non-international students. Students were not asked to identify their sex upon $\mathrm{CCW}$ registration.

From the feedback forms, approximately $50 \%$ of student participants reported that their main reason for attending was to make new friends while the other $50 \%$ reported that they attended to learn how to cook nutritious meals. Ninety percent of students reported making a new friend from the workshop. A majority of students reported that they were confident that they could incorporate ideas/tips/strategies from the workshop into their lives $(94 \%)$, while $85 \%$ of students reported that their knowledge/understanding about meal preparation improved because of the session. Finally, $84 \%$ of students reported that their motivation or intention to improve their nutrition habits increased as a result of the workshop and $76 \%$ of students reported that their knowledge/understanding about healthy eating improved as a result of the workshop.

In the open-ended feedback form questions, the majority of students reported that socializing with others was their favourite part of the workshops. Other highlights of the workshops included preparing and making the food, meeting new people, and the friendly and welcoming atmosphere of the workshops. The most common suggestion to improve the workshops related to providing different recipes (desserts and main dishes) 
and cuisines, followed by holding the workshops in a larger space, and presenting additional nutrition information.

\section{Qualitative Data: Semi-Structured Interviews}

The key questions asked to the student participants in the semistructured interviews and their answers are presented below.

\section{Why did you participate?}

When participants were asked why they decided to participate in the workshops, the two most frequent answers were to meet new people and to learn something new about cooking/western cuisine. Other reasons included that the event was free, food was provided, cooking was an activity that interested the participant, and the participant wanted to learn a specific recipe.

I'm interested in cooking with others and ... in this workshop you guys provided all the ingredients, many of them that I have never seen before... this is my reason, to... have to gain more experience about cooking western food, and second ... I can meet ... more people make some friends in this workshops.

\section{What did you learn?}

All participants indicated that they learned something new about nutrition and cooking after attending a workshop regardless of their existing nutritional and cooking abilities and knowledge. The biggest learning outcome was the understanding that food should be consumed in balance and in moderation. Many students highlighted and referenced the Eat Well Plate graphic that is utilized at the workshop to teach participants about the recommended proportion of vegetables (half a plate), grains (a quarter of the plate), and protein (a quarter of the plate) according to the Government of Canada (Government of Canada, 2016). Participants also learned that consuming foods labelled as "less healthy", such as those containing sugar, in moderation, is okay. Other learning outcomes included learning how to

- work collaboratively with others,

- cut vegetables,

- measure ingredients,

- use North American measuring tools,

- bake,

- use kitchen appliances, such as an oven, and

- read and follow recipes. 
Students also reported becoming more familiar with North American food and how to prepare it, gaining information about healthy eating, such as how to make sugar substitutions, and shopping cost effectively, such as selecting frozen over fresh fruit. One student summarized her learning at the cooking workshops as obtaining life skills.

I learned that it is not just fruits and vegetables, it's kind of like portioning of things.... like when I have rice I have rice with like some...carrot things...

I'm learning how to prepare those foods, I didn't know how to prepare those food before, like peppers how you cut peppers in that way.

\section{Did you see any behaviour changes after participating?}

All interviewed students mentioned behaviour changes as a result of participating in the workshop. In many cases students reported that what they had learned translated to a change in their eating behaviours. In particular, students noted that the workshops increased their awareness for a balanced diet and incorporated more fruits and vegetables into their diets.

What I'll do is ... I'll buy carrots kind of things and put it in my rice ... cause the way they put it like this is like half of your plate should be fruit and veg...

Other behaviour changes included purchasing of foods that were presented in the workshop because of increased familiarity with those items.

I didn't use onions before participating in this cooking workshop...every time I cut it I cry...So I just don't buy them. But then after the workshop I started using them.

So in the workshop I saw stuff and because this stuff is now familiar to me I go to mall and buy this stuff when I shop.

Students noted a variety of changes related to the way they cooked, which included beginning batch cooking to save time, purchasing frozen fruits, mixing fruit with yogurt, and becoming more flexible in their cooking, all of which was demonstrated at the cooking workshops. Participants indicated purchasing new cooking utensils that they were introduced to through the workshops, such as a blender.

I bought a smoothie blender... 'cause I like that smoothie taste and I also bought I used to drink yogurt but I've never tried yogurt with fruits... I bought all those stuff and do it at my 
home...And I think oh that's cool I like it and it's really healthy ....so I like the idea it makes me thinking about I can buy something really simple... to support my eating habit.

When I cook I remember the workshop and I try to organize everything in a way it was organized during the workshop.... have separate bowls for different ingredients chop it before.

The largest barriers for participants to incorporate what they had learned at the workshops into their cooking practices was the lack of cooking utensils, and difficulty leaving familiar ethnic cuisines for more North American cuisine.

Those kind of stuff I would try it back home cause I don't have those how you say that?.. the blender. I don't have that.

I still prefer the Chinese food even though ... the food we cook in the cooking shop are much healthier ... [but] I still eat Chinese food when I'm cooking at home ...that's just familiar.

Interestingly, not all behaviour changes were food related. Students discussed how the workshops helped them to build social skills, meet new people, and keep in contact with peers in the same academic program. One student discussed how the workshops helped her to become more comfortable in new social situations.

Generally the people um in fact before I before I came here [cooking workshop] I was generally a very introvert person and I didn't I preferred to staying alone ... but now it's I feel much better.. I'm always trying to put myself out of my comfort zone and I think I also I think I did it.

\section{What is healthy eating and how did the cooking workshops influence it?}

Students were asked to provide their definition of healthy eating. Their definitions of healthy eating included eating that is tailored to the energy requirements of an individual based on their activity levels, that varies from person to person, that consists of a balanced diet of fruits, vegetables, dairy, and meat products with all types of food eaten in moderation and no food consumed in excess.

It's food that good for your health, you know it doesn't contain any ingredients that can somehow damage you, and this food is tasty and it allows you to preform activities that you need, .... So 
healthy food varies from a person to person. What is healthy for one can be damaging for another.

The workshops served as a reminder to frequently incorporate fruits and vegetables in their diet and emphasized the importance of eating all types of foods in balance and moderation. Cooking workshop participants most commonly stated that the workshop helped to refine their definition of healthy eating.

It changed [my definition of healthy eating] because before that I thought that if a food is really healthy, it's not as tasty as a regular food. But here I realized that you don't have to be so strict and passion about ingredients. So sugar is fine in moderation, flour is fine it's just the important thing is to find a balance between.

\section{What did you like about the workshops?}

Participants most frequently commented on how they enjoyed the friendly social atmosphere of the workshops, as it provided them an opportunity to cook with others, relax, talk with other international students, meet students from various majors and walks of life, and practice their English.

I also enjoyed the whole process... When you do it, you feel yourself like a part of the community...And this what I really enjoyed.

So I think that's what I really like about this cooking workshop is something that learning is enhanced when you have people around.

...It was fun mostly because I was really stressed and it was a good time...good break with a good bunch of people.

...we don't have a lot of opportunities to meet all the exchange or international students I feel like this is a great opportunity for you to meet new friends.

Students also noted that they enjoyed the opportunity to learn new recipes, and to cook and taste Canadian cuisine. Participants commented on the informative nutrition presentations, and the professional execution of the workshops, providing clear instructions and assistance when needed. Students liked receiving the emailed versions of the recipe guides so that they could try these new recipes at home. 
I just thought it was informative ... I thought there was a lot to take about from it and I just love getting new recipes... [it] pushes me out of my comfort zone instead of just going to my go to.

My favourite parts is we can cook all kinds of different kinds of food each time and not only we can cook together you show us you show us the instructions very very detailed and we can get a recipe home.

I feel like this workshop ...would help me cause I know where to find the information about the nutrition ... I think it's helpful.

\section{How can we improve future workshops?}

Participants contributed many ideas for improving future cooking workshops. The most commonly suggested improvement was to incorporate different ethnic cuisines. Participants were keen to learn about different cultures' cuisines, and discussed how the cooking workshops provided an optimal environment to expand their taste palate. Participants explained how the cooking workshops were a part of their cultural experience as an international student and provided them with a unique opportunity to experience different foods that they would not get the opportunity to taste and prepare back home. Students suggested having themed cooking workshops where the menu would be designed around a certain country's cuisine, and to have international students of that nationality share with other participants their cultural dishes, and even take a lead in the workshops.

We can try all kinds of cuisine from other countries... [it] is a good opportunity for us to attend this workshop to try things that we've never tried before. That's very unforgettable experience.

Students emphasized that the recipes used in the cooking workshop should contain few and simple ingredients and be as easy and quick to cook as possible. It was also suggested that workshops could be themed around the use of one particular ingredient, demonstrating recipe modification or how to use basic cooking tools. Additionally, students suggested more frequent workshops as registration filled quickly for these events, and to acquire a larger cooking space with modern cooking appliances. Other students expressed an interest in incorporating more nutrition information as part of the cooking workshops. Participants mentioned the use of YouTube and interactive quizzes and games as part of the nutrition information 
sessions. Students suggested additions to the workshop in food literacy, for example teaching how to read food labels, certain ingredients' and nutrients to look for, and understanding food "buzz" words used in the marketing of food such as "organic" and "GMO." Participants showed an interest in learning about the health benefits of certain fruits and vegetables, and why you would consume them. One student suggested that providing new international students with the nutrition information which was presented at the workshops and/or hosting cooking workshops for these students, could help ease their transition to the new environment.

Students noted that oftentimes international students of similar cultures would congregate together, and so by encouraging students to create new diverse groups, it could help challenge international students to meet new people, learn about new cultures, and practice their English.

I really like the idea of gathering together and sharing our experience um sometimes I found out people from the same culture would just instantly get together but maybe you can challenge people...so in this way people can have communication with different culture.

\section{DISCUSSION AND CONCLUSIONS}

The cooking workshop outcomes have been two-fold: critical nutrition information is being provided to international student participants and nutrition behaviour change is reported, and participants are experiencing an enhanced sense of community and social connection. By equipping students with the necessary nutrition skills to cook for themselves, and make informed food decisions, the CCWs provide a cost-effective, interactive approach for international students to gain cooking skills in a supportive environment and contribute to behaviour changes that extended beyond the workshop itself. Most notably, the CCWs provide a low barrier opportunity for international students to meet other students and increase their social networks to protect against isolation, which is very common in the international student population (Almohanna, Conforti, Eigel, \& Barbeau, 2015). Students in this study noted that among common challenges faced by international students, language barriers and the negative effects of isolation were most influential. The CCWs have a positive impact on the social experiences of these vulnerable students.

The continued use of the peer-to-peer interaction is especially integral in supporting the nutritional well being of international students who may be in need of sound nutrition education to improve their eating patterns while 
exploring a new-found independence. The peer-led approach allows for student participants to connect with current students and creates a safe space for discussion on sensitive health topics such as substance use and body image. The learning environment created at the workshops has been especially effective in that student leaders facilitate this peer-to-peer support and learning through experiential means. Considering the barriers to accessing social activities for international students on campus, the cooking workshops have provided a social outlet wherein students can meet other students in a welcoming, engaging, and supportive social setting. The cooking workshops also provided students with a cultural experience to enjoy some Canadian cuisine.

\section{IMPLICATIONS}

Our findings support previous literature on cooking workshops for postsecondary students (Ball \& Brown, 2012; Clifford, Anderson, Auld \& Champ, 2009; Lawe, 2013; Levy \& Auld, 2004). Similar to these research findings, after participating in the CCWs, students noted increased nutrition, food preparation skills and healthy eating knowledge, intention to change their nutrition behaviours and increased cooking skill self-efficacy.

While this was not a randomized control trial, this study has demonstrated that the workshops are having positive short-term impacts on the cooking and nutrition behaviours of international students. Future research should consider a randomized control trial to quantify the behavioural outcomes of the cooking workshops. It would be of value to assess the long-term impacts of the workshops on overall diet quality and cooking skills, and social connection, and whether students continue to use the skills and information learned in the workshops long after participating.

Improvements for future workshops include securing a larger space and exploring new ways to introduce students to new ethnic cuisines, while keeping nutrition education at the core of these cooking workshops. Facilitating these workshops in a larger kitchen space could allow for the workshops to host more students and increase the positive impact on students' social and nutritional wellbeing. One continued partnership that could be strengthened is with SFU International Services for Students, to identify if it would be possible to provide additional funding and integrate the CCW program or a shorter nutrition intervention into an orientation for international students. By allowing these workshops to be an experience that every international student has upon arriving to SFU, it could help international students meet each other and create a community amongst this 
population, while teaching students valuable skills that would serve them while studying at SFU and beyond.

With the increased use of online platforms, it is no surprise that emerging nutrition interventions focus on utilizing online platforms to engage students. The use of randomized controlled trials assessing the effectiveness of email and text updates to post-secondary students about nutrition information have shown promising results (Brown, O'Connor, \& Savaiano, 2014; Mackey, Schweitzer, Hurtado, Hathway, DiPietro, Lei, \& Klein, 2015). Post-secondary students reported being receptive to using these online resources (Mackey et al., 2015) and students reported improved nutrition knowledge, and in some cases behaviour modifications such as increased fruit and vegetable consumption (Brown, O'Connor, \& Savaiano, 2014). Future workshops may want to consider incorporating an online/text component post workshop to keep students engaged and informed about nutrition information. This post workshop interaction may encourage students to continue to make healthy nutrition choices and may contribute to long-term nutrition behaviour changes as a result of the workshops.

Many students who were interviewed identified barriers to incorporating what they learned from the workshops into their daily lives. University-wide strategies such as bulk food purchasing, food recovery programs, and food subsidies, should be considered to improve accessibility to healthy, affordable, and ethnically appropriate food, to enable students to easily incorporate the skills and knowledge learned from the CCWs into their daily lives. Future research should seek to understand how post-secondary institutions can be used as a setting for health promotion to support the physical, emotional, and academic needs of international students.

Given the outcomes of the CCWS for international students, these workshops could also be impactful for local students. Considering the demands of university life, along with the level of independence that comes with the university student experience, there is value in learning life skills such as food preparation techniques and basic nutrition information. Many students will transition to the workforce or pursue graduate studies. Educating students on nutrition within the post-secondary setting, can serve as an effective method to support overall student wellbeing. By incorporating domestic students into the workshops, we might also provide opportunities for international students to integrate into the wider campus community to offset psychosocial and acculturative stress. 


\section{REFERENCES}

Alakaam, A., Castellanos, D., Bodzio, J. \& Harrison, L (2015). The Factors That Influence Dietary Habits Among International Students in the United

States. Journal Of International Students, 5(2): 104-120. doi:10.1080/07359680802619834.

Almohanna, A., Conforti, F., Eigel, W., \& Barbeau, W. (2015). Impact of Dietary Acculturation on the Food Habits, Weight, Blood Pressure, and Fasting Blood Glucose Levels of International College Students. Journal of American College Health, 63(5), 307-314. doi:10.1080/07448481. 2015.1025075.

American College Health Association (ACHA). American College Health Association-National College Health Assessment II: Canadian Reference Group Executive Summary Spring 2016. Hanover, MD: American College Health Association; 2016.

Ball, B., \& Brown, L. B. (2012). Qualitative Description of College Students Dinner Groups. Journal of Nutrition Education and Behavior, 44(1), 29-35. doi:10.1016/j.jneb.2011.04.008.

Brown, O. N., O’Connor, L. E., \& Savaiano, D. (2014). Mobile MyPlate: A Pilot Study Using Text Messaging to Provide Nutrition Education and Promote Better Dietary Choices in College Students. Journal of American College Health, 62(5), 320-327. doi:10.1080/07448481.2014.899233.

Clifford D., Anderson J., Auld G. \& Champ J. (2009). Good Grubbin': impact of a TV cooking show for college students living off campus. Journal of Nutrition Education and Behavior, 41(3), 194-200. doi:10.1016/j.jneb. 2008.01.006.

Deitians of Canada. (2012). Promoting Mental Health through Healthy Eating and Nutritional Care. Retrieved from https://connect.sfu.ca/service/home/ $\sim /$ ?auth $=$ co\&loc $=$ en_US\&id $=68629 \&$ part $=3 \&$ view $=$ html.

Government of Canada (2016). Build a healthy meal: use the Eat Well Plate. Retrieved from http:/www.healthycanadians.gc.ca/eating-nutrition/ healthy-eating-saine-alimentation/tips-conseils/interactive-tools-outilsinteractifs/eat-well-bien-manger-eng.php.

Lawe, B. (2013). Teaching university students to cook, to improve their diet: a pilot study at Nottingham Trent University. International Journal of Health Promotion and Education, 51(3), 161-168. doi:10.1080/14635240.2012.758884.

Levy, J., \& Auld, G. (2004). Cooking Classes Outperform Cooking Demonstrations for College Sophomores. Journal of Nutrition Education and Behavior, 36(4), 97-203. doi:10.1016/s1499-4046(06)60234-0.

Mackey, E., Schweitzer, A., Hurtado, M. E., Hathway, J., Dipietro, L., Lei, K. Y., \& Klein, C. J. (2015). The Feasibility of an E-mail-Delivered Intervention to Improve Nutrition and Physical Activity Behaviors in African American 
College Students. Journal of American College Health, 63(2), 109-117. doi:10.1080/07448481.2014.990971.

Smith, R., \& Khawaja, N. (2011). A review of the acculturation experiences of international students. International Journal of Intercultural Relations, 35(6), 699-713. doi:10.1016/j.ijintrel.2011.08.004

Simon Fraser University Institutional Research and Planning (SFU IRP). (2017). Fall 2017 International Student Report. Retrieved from http://www.sfu.ca/ content/dam/sfu/irp/students/visa_report/visa.report.1177.pdf.

Thorpe, M. G., Kestin, M., Riddell, L. J., Keast, R. S., \& Mcnaughton, S. A. (2013). Diet quality in young adults and its association with food-related behaviours. Public Health Nutrition, 17(08), 1767-1775. doi:10.1017/ s1368980013001924.

Versaevel, L. N. (2014). Canadian Post-Secondary Students, Stress, and Academic Performance-A Socio-Ecological Approach. Electronic Thesis and Dissertation Repository. Paper 2657.

White, S., Park, Y. S., Israel, T., \& Cordero, E. D. (2009). Longitudinal Evaluation of Peer Health Education on a College Campus: Impact on Health Behaviors. Journal of American College Health, 57(5), 497-506. doi:10.3200/jach.57.5.497-506.

Zhang, J., \& Goodson, P. (2011). Acculturation and psychosocial adjustment of Chinese international students: Examining mediation and moderation effects. International Journal of Intercultural Relations, 35(5), 614-627. doi:10.1016/j.ijintrel.2010.11.004

GABRIELLA LUONGO, is a Master's of Public Health (Health Promotion) candidate at the University of Toronto. She received her BSc (Hons) in Health Sciences from Simon Fraser University where she was a Peer Health Educator and lead facilitator of the Community Cooking Workshops from 2014-2016. Her major research interests lie in food policy, nutrition program implementation and evaluation, and understanding how post-secondary institutions can be used as a setting for health promotion. Email: g.luongo@mail.utoronto.ca

ROCHELLE TUCKER, DSc, is a Senior Lecturer in the Faculty of Health Sciences at SFU. Her major research interests lie in research methodology, mental health and adolescent development. Part of Dr. Tucker's research is focused on understanding gender, ethnic and socio-economic disparities in adolescent mental health, and the relationships between mental health and other health issues such as nutrition, and obesity in adolescence. She is currently working on integrating an online formative teaching inquiry tool to enhance students' learning and instructors' teaching. Email: tucker@sfu.ca

CRYSTAL HUTCHINSON, MEd, CHES, is a Workplace Wellbeing Strategist at the University of British Columbia where she is leading strategic and 
comprehensive University-wide workplace health promotion actions and initiatives in partnership with faculties, departments and administrative units. In her previous role as Health Promotion Specialist at Simon Fraser University (SFU), Crystal worked to advance the Healthy Campus Community initiative. Her work at SFU included the supervision and evaluation of the Peer Health Education program from 2013-2017. Email: crystal.hutchinson@ubc.ca

ROSIE DHALIWAL, MEd, RD, is the Acting Associate Director, Health Promotion at SFU Health and Counselling Services. She has diverse experiences in nutrition and dietetics including roles as a Clinical Dietitian and Nutrition Consultant in the community, also Distance Education Supervisor and Sessional Instructor at SFU. She has dedicated her clinical and educational expertise to the Health Promotion team at SFU since 2007 where she trains and mentors the Peer Health Educators and leads the award winning Healthy Campus Community initiative. Email: rosie_dhaliwal@sfu.ca 